Artículo de revisión

\title{
Salud bucal en sujetos con trastorno del espectro autista: consideraciones para la atención odontológica
}

\section{Oral health in subjects with autistic spectrum disorder: considerations for dental care}

\section{Saúde oral em sujeitos com transtorno do espectro autístico:} considerações para assistência odontológica

Lesbia Rosa Tirado- Amador ${ }^{1 凶} \underline{\mathrm{ORCID}}$, Meisser Madera ${ }^{2 凶} \underline{\mathrm{ORCID}}$, Carlos Arturo Leal-Acosta ${ }^{3 凶} \underline{\mathrm{ORCID}}$

${ }^{1}$ Odontóloga. Especialista en Estadística Aplicada. Profesor coordinador de investigaciones y líder del grupo GINOUS, Escuela de Odontología, Universidad del Sinú seccional Cartagena.

2 Ph.D en Metodología de Investigación Biomédica y Salud Pública. Profesor Facultad de Odontología, Universidad de Cartagena. Grupo de Investigación en Salud Pública (GISPOUC).

${ }^{3}$ Odontólogo, Magister en microbiología clínica. Profesor Programa de Odontología, Corporación Universitaria Rafael Núñez. Grupo de Investigación en salud CURN (GISACC).

Fecha correspondencia:

Recibido: septiembre de 2020.

Aprobado: febrero de 2021.

\section{Forma de citar:}

Tirado- Amador LR, Madera M, Leal-Acosta CA. Salud bucal en sujetos con trastorno del espectro autista: consideraciones para la atención odontológica. Rev. CES Odont 2021; 34(2): 139 158. https://doi.org/10.21615/ cesodon. 5846

Open access

(C) Derecho de autor Licencia creative commons Ética de publicaciones Revisión por pares Gestión por Open Journal System DOI: 10.21615/cesodon.5846

\section{Resumen}

El estado de salud bucal y la atención odontológica en sujetos con Trastornos del Espectro Autista (TEA), amerita atención, dada las características específicas en su fisiopatología bucal y algunos factores comportamentales que influyen el progreso de enfermedades bucales como la caries dental y la enfermedad periodontal. El objetivo de la presente revisión fue describir la evidencia referente a salud bucal, así como consideraciones relevantes para la atención odontológica en sujetos con TEA. Se realizó búsqueda sistemática de la literatura empleando los buscadores de información: EBSCOhost, ScienceDirect, y Scopus. Los descriptores empleados fueron: Trastorno del Espectro Autista, Salud Bucal, Caries dental, Enfermedades Periodontales, Atención Odontológica (DeCS-Bireme), sus combinaciones y equivalencias en inglés Autism Spectrum Disorder, Oral health, Dental caries, Periodontal Diseases, Dental care (MeSH-Database). Considerando artículos publicados entre 2014 y septiembre de 2019. Inicialmente, se revisaron los títulos y aplicaron criterios 
ISSNe 2215-9185

ISSN 0120-971X de elegibilidad para la descripción de evidencia incluida, correspondiente a 46 artículos, considerando su enfoque, tipología, idioma y año de publicación y luego se plantea un análisis de la información. Concluyendo que la evidencia sigue siendo controversial y limitada con tendencia a destacar

deficiencias en la higiene bucal que se realiza en los hogares de sujetos con TEA, la presencia de caries dental, enfermedad periodontal y algunos hallazgos que condicionan la fisiología oral y progreso de enfermedades bucales. Así mismo, se resalta la dificultad en la atención odontológica a causa de la cuestionable preparación del personal de odontología y los consecuentes temores en los padres que puede limitar la asistencia a la consulta de manera oportuna y en edades tempranas para lograr una buena adaptación. Por todo lo anterior, la atención odontológica se convierte en un desafío que deberá orientar sus acciones con empatía para atender esta población con participación de la familia, profesores y otros miembros de instituciones especializadas, con carácter holístico y de medicina familiar.

Palabras clave: trastorno del espectro autista; salud bucal; enfermedades de la boca; padres; cuidadores. (Fuente DeCS-Bireme).

\section{Abstract}

Oral health status and dental care in subjects with Autism Spectrum Disorders (ASD) deserves attention, given the specific characteristics of their oral pathophysiology and some behavioral factors that influence the progress of oral diseases such as dental caries and periodontal disease. The objective of the present review was to describe the evidence regarding oral health as well as relevant considerations for dental care in subjects with ASD. Therefore, a systematic search of the literature was carried out using the information seekers: EBSCOhost, ScienceDirect, and Scopus. The descriptors used were: Trastorno del Espectro Autista, Salud Bucal, Caries dental, Enfermedades Periodontales, Atención Odontológica (DeCS-Bireme), their combinations and equivalents in English: Autism Spectrum Disorder, Oral health, Dental caries, Periodontal Diseases, Dental care (MeSH-Database). Considering articles published between 2014 and September 2019. Initially, the titles were reviewed and eligibility criteria were applied for the description of the included evidence, corresponding to 46 articles, considering their focus, typology, language and year of publication, and then an analysis of the information was proposed. Concluding that the evidence remains controversial and limited, with a tendency to highlight deficiencies in oral hygiene performed in the homes of individuals with ASD, the presence of dental caries, periodontal disease, and some findings that condition oral physiology and the progress of oral diseases. Likewise, the difficulty in dental care is highlighted due to the 
questionable preparation of the dental staff and the consequent fears in parents that may limit attendance at the consultation in a timely manner and at an early age to achieve a good adaptation. Due to all the above, dental care becomes a challenge that should guide its actions with empathy to serve this population with the participation of the family, teachers and other members of specialized institutions, with a holistic and family medicine nature.

Keywords: autism spectrum disorder; oral health; mouth diseases; parents; caregivers (Source MeSH-Database).

\section{Resumo}

O estado da saúde bucal e da assistência odontológica em indivíduos com Transtornos do Espectro do Autismo (TEA) merece atenção, dadas as características específicas de sua fisiopatologia bucal e alguns fatores comportamentais que influenciam no progresso de doenças bucais, como cárie dentária e doença periodontal. O objetivo da presente revisão foi descrever as evidências sobre a saúde bucal, bem como considerações relevantes para o cuidado odontológico em indivíduos com TEA. Portanto, uma busca sistemática da literatura foi realizada usando os motores de busca de informações: EBSCOhost, ScienceDirect e Scopus. Os descritores usados foram: Trastorno del Espectro Autista, Salud Bucal, caries dental, Enfermedades Periodontales, Atención Odontológica (DeCS-Bireme), suas combinações e equivalentes em inglês Autism Spectrum Disorder, oral health, dental caries, Periodontal Diseases, dental care (MeSH-Database). Considerando os artigos publicados entre 2014 e setembro de 2019. Inicialmente, os títulos foram revisados e os critérios de elegibilidade aplicados para a descrição das evidências incluídas, correspondendo a 46 artigos, considerando seu foco, tipologia, idioma e ano de publicação, para então uma análise é proposta das informações. Concluindo que as evidências permanecem controversas e limitadas, com tendência a evidenciar deficiências na higiene bucal realizada nas residências de indivíduos com TEA, a presença de cárie dentária, doença periodontal e alguns achados que condicionam a fisiologia oral e o progresso das doenças bucais. Da mesma forma, destaca-se a dificuldade no atendimento odontológico devido ao preparo questionável da equipe odontológica e os consequentes temores dos pais que podem limitar o comparecimento à consulta em tempo hábil e em idade precoce para uma boa adaptação. Por tudo isso, a assistência odontológica torna-se um desafio que deve nortear suas ações com empatia para atender essa população com a participação da família, professores e demais membros de instituições especializadas, com caráter holístico e de medicina de família. 
Palavras chave: transtorno do espectro autista; saúde bucal; doenças da boca; pais; cuidadores. (Fonte DeCS-Bireme).

\section{Introducción}

El estado de salud bucal es relevante al influir sobre la salud general, debido a que la cavidad bucal interviene en múltiples actividades biológicas con la masticación, alimentación, comunicación y el establecimiento de relaciones sociales (favoreciendo la autoestima) ${ }^{(1)}$. Algunos síndromes y trastornos del desarrollo neurológico, como el trastorno del espectro autista (TEA), representan una condición desafiante para los Especialistas en Psicología, Pedagogía y Medicina ${ }^{(2)}$. De acuerdo con el manual de los trastornos mentales (DSM-5) los TEA incluyen los diagnósticos de trastorno autista, trastorno de Asperger, trastorno desintegrativo de la infancia y trastorno generalizado del neurodesarrollo ${ }^{(3)}$, cuyo nivel de inteligencia e interacción social, pueden variar en diversos grados, condicionando la asistencia y prestación de los servicios de salud.

Hasta hace poco, la información sobre la epidemiología del autismo se basó en estudios de la infancia considerando el fenotipo de autismo severo y circunscrito en áreas geográficas pequeñas ${ }^{(4,5)}$. Un reciente ascenso en sus cifras evidencia cuatro o cinco de cada 10.000 niños nacidos vivos, afectando más a hombres en proporción 3:1 (1), su prevalencia se estima con valores que oscilan entre 0,7 y $1,5 \%$ en infancia y adolescencia, considerando principalmente aquellos sin discapacidad intelectual comórbida, estas variaciones podrían obedecer al diseño metodológico o enfoque de los estudios ${ }^{(6,7)}$.

Para el profesional de la odontología, el TEA puede ser una condición desconocida, que amerita interés pues puede condicionar la evolución de condiciones bucales, requiriendo acceder al estado actual del conocimiento en salud bucal y atención odontológica para esta población. Por tanto, el objetivo de esta revisión es identificar y describir la evidencia en personas con TEA sobre su salud bucal, caries y enfermedades periodontal, presencia de hábitos orales (bruxismo entre otros) y las consideraciones durante la atención odontológica, sus comportamientos y los roles que pueden desempeñar quienes rodean a estos pacientes.

\section{Estrategia de búsqueda}

Se realizó una búsqueda sistemática entre septiembre y octubre del 2019, en tres bases de datos electrónicas: EBSCOhost, ScienceDirect, Scopus, utilizando las palabras clave Trastorno del Espectro Autista en combinación con Salud Bucal, Caries dental, Enfermedades Periodontales, Atención Odontológica y en inglés sus equivalencias Autism Spectrum Disorder 
en combinación con Oral health, Dental caries, Periodontal Diseases, Dental care. El conector para conjugación de términos fue " $\mathrm{y}$ " y su equivalencia en inglés "And". Se consideraron las publicaciones de estudios realizados en el periodo de 2014 a 2019, en idioma español e inglés. Se realizó una evaluación preliminar, considerando en el título la combinación de los descriptores o su sinonimia, se eliminaron las duplicaciones, consiguiendo 55 artículos. Posteriormente 2 revisores aplicaron criterios de selección a partir de los resúmenes, logrando un acuerdo de 0,85 (prueba Kappa Cohen), luego mediante un panel de discusión, se logró consenso para la aceptación de 46 artículos. Para su inclusión, se consideraron algunos criterios de cumplimiento en investigación primaria como la claridad en la procedencia de los sujetos de estudio, muestra superior a 10 individuos, criterios de selección y declaración de aspectos éticos. En caso de Investigación secundaria (revisiones de literatura), se consideró la declaración de objetivo y tópico de estudio (con mínimo 20 referencias). La figura 1, ilustra el número de artículos incluidos finalmente en esta revisión. Fueron excluidos boletines y reportes de casos clínicos.

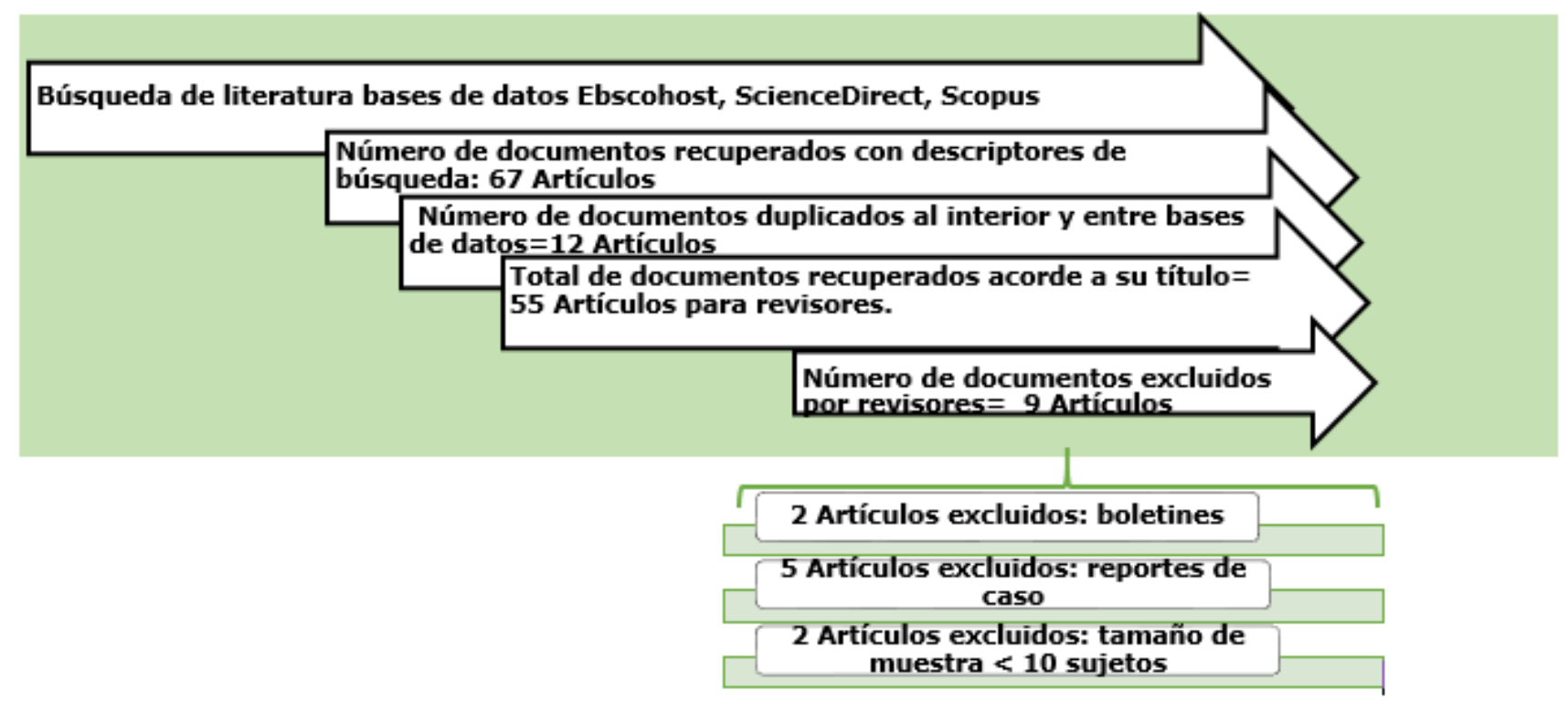

Figura 1. Flujograma de búsqueda para obtención de artículos insumo de revisión. 


\section{Desarrollo}

\section{Resultados de la búsqueda y características de estudios seleccionados}

En los 46 estudios seleccionados, predominaron los artículos descriptivos, seguido de revisiones de literatura (Tabla 1). Respecto al idioma de publicación, 42 artículos se encontraban en inglés y 4 en español. Los años en los cuales se encontró mayor número de publicaciones fueron 2015 y 2017 aportando conjuntamente 39,1\% de los artículos incluidos.

Tabla 1. Cantidad de artículos según enfoque y tipología.

\begin{tabular}{lcc}
\hline \multicolumn{1}{c}{ Enfoque Investigativo } & Tipo de publicación & $\begin{array}{c}\text { Cantidad y \% de } \\
\text { publicaciones según tipología } \\
\text { en los artículos seleccionados }\end{array}$ \\
\hline $\begin{array}{l}\text { Investigación primaria } \\
\text { cuantitativa }\end{array}$ & Estudios descriptivos & $14(30,4)$ \\
\hline $\begin{array}{l}\text { Investigación primaria } \\
\text { cuantitativa }\end{array}$ & Estudios analíticos & $11(23,9)$ \\
\hline $\begin{array}{l}\text { Investigación primaria } \\
\text { cuantitativa }\end{array}$ & $\begin{array}{c}\text { Estudios } \\
\text { experimentales }\end{array}$ & $7(15,2)$ \\
\hline $\begin{array}{l}\text { Investigación primaria } \\
\text { cualitativa }\end{array}$ & Estudios cualitativos & $3(6,5)$ \\
\hline Investigación secundaria & Revisiones de literatura & $11(23,9)$ \\
\hline & $\boldsymbol{n}=46(100 \%)$ & \\
\hline
\end{tabular}

\section{Hábitos de higiene bucal, caries dental y estado periodontal}

En personas con TEA, se ha reportado higiene oral deficiente ${ }^{(8)}$, lo que quizás está relacionado con la presencia de limitación motora, afectando negativamente la realización del cepillado dental ${ }^{(9)}$, por lo cual, se recomienda la asistencia y supervisión de padres y cuidadores ${ }^{(3)}$, evitando así intervenciones odontológicas complejas e innecesarias que aumentan la ansiedad en estos pacientes. Otras posibles explicaciones, son la retención de los alimentos entre las mejillas, el rechazo por el sabor de la crema dental ${ }^{(10)}$, la baja frecuencia de cepillado dental o la existencia de una rutina de cepillado con mucha variabilidad durante el día, lo que facilita la omisión en el uso de seda dental y cepillado nocturno y ${ }^{(11)}$. En un estudio realizado en Hong Kong ${ }^{(12)}$, en 190 pacientes (casos confirmados y sospechosos de TEA), se muestra que al menos un 30\% de los niños tenían hábitos de cepillado una vez al día, situación que puede convertirse en un factor para perpetuar la permanencia de residuos alimenticios y placa bacteriana en tejidos duros y blandos. 
La evidencia disponible, referente a la presencia y severidad de caries dental en sujetos con TEA, resulta controversial, algunos estudios reportan una prevalencia de caries similar en comparación a controles e incluso menor en sujetos con TEA ${ }^{(3,8,11,13)}$. Lo anterior discrepa de los estudios realizados en Indonesia ${ }^{(14)}$ y Pakistán ${ }^{(15)}$, en donde la prevalencia de caries fue alta en sujetos con TEA. Esta variabilidad en los resultados, puede tener diversos orígenes. Por un lado, las variaciones metodológicas, la heterogeneidad de las muestras o diseños de estudio que no permiten la confirmación de una hipótesis causal ${ }^{(1)}$. Así mismo, la existencia de limitados estudios de casos y controles referentes al estado de la salud bucal en pacientes con TEA, precisamente una revisión sistemática realizada por Bartolomé en 2016, solo reporto 10 estudios ${ }^{(16)}$, lo que indica una publicación limitada hasta esa fecha. La heterogeneidad en los resultados, también puede estar influenciada por la edad de los pacientes con TEA, las condiciones socioculturales y el nivel educativo de los pacientes y de sus padres, la categoría y severidad del diagnóstico del síndrome, así como a la conducta y comportamiento evidenciado en la vida cotidiana de quien padece el síndrome, en donde la presencia de estereotipias motoras simples ${ }^{(8,9)}$, podrían ser incluidas en la rutina de cepillado, provocando una optimizando en la higiene bucal o favoreciendo un cepillado traumático.

Pese a la discusión en cuanto a la frecuencia de caries dental, existe concordancia entre los factores condicionantes para incrementar el riesgo de caries. Uno de estos, corresponde al uso de fármacos estimulantes del sistema nervioso central, antidepresivos, antihipertensivos, anticonvulsivantes y antipsicóticos, aunque existe falta de hipótesis para estos hallazgos ${ }^{(3)}$, se ha reportado que algunos fármacos afectan la fisiología bucal y autoclisis salival, pues incluyen edulcolorantes y otros favorecen la disminución y composición del flujo salival, disfagia, disgeusia, sialoadenitis y glositis $(10,11,17)$.

Algunos estudios alusivos referentes al estado periodontal en pacientes con TEA, lo reportan como deficiente y se enfocan en los hábitos cuestionables de higiene bucal ${ }^{(3,8,9)}$. En tanto, se han reportado positivamente algunos marcadores de salud periodontal en presencia de TEA (18). Un hallazgo periodontal frecuentemente reportado, son las recesiones gingivales vestibulares, posiblemente asociadas bien sea con la condición periodontal fisiológica, el cepillado dental sin control y/o agresivo a causa de episodios autolesivos, los cuales son comunes en TEA ${ }^{(10,15)}$, o también relacionados con la presencia de hábitos parafuncionales y maloclusiones ${ }^{(11)}$. En un estudio realizado en Libia, de $2015^{(19)}$, existen notables necesidades de tratamiento periodontal para pacientes con TEA, afirmando un campo de oportunidad para realizar intervención mediante prácticas de autocuidado e higiene bucal para lograr la reducción de necesidades de tratamiento profesional. 
En referencia a lo anterior, a la luz de los autores, son limitadas las publicaciones alusivas a periodontitis y estado periodontal en sujetos con TEA, quizás debido a la edad de los sujetos valorados ${ }^{(1)}$, siendo importante realizar estudios longitudinales en diversos grupos etarios.

\section{Características intraorales y extra orales de interés odontológico}

Existen algunos hallazgos orofaciales en pacientes con TEA, los cuales no pueden considerarse patognomónicos para esta población, pues podrían encontrarse en cualquier alteración mental o física e incluso en ausencia de ambas; sin embargo, revisten interés odontológico para realizar diagnósticos desde la consulta, destacando así la presencia de paladar hendido y profundo, mordida abierta anterior, mordida cruzada posterior, apiñamiento dental, glositis migratoria, lengua fisurada, lengua saburral, inserción inadecuada de frenillos labiales y linguales, alteraciones del desarrollo dental, presencia de facetas de desgaste e incidencia de hábitos como bruxismo, onicofagia, queilofagia, mordedura de objetos (pica, grava, colillas de cigarrillo, bolígrafos ), regurgitación repetitiva y retención de comida en mejillas $(8,9,20)$.

La presencia de traumatismos dentales, es frecuente con cifras que oscilan entre $70 \%$ a $75 \%$, destacando los golpes en la cara, cabeza, rasguños en encías, queilofagia y ulceras traumáticas ${ }^{(3,9)}$, por actividades rutinarias o por episodios autolesivos en los pacientes con TEA ${ }^{(10,15)}$. En este sentido, los traumatismos dentales se tornan interesantes, pues pueden ocasionar daño a largo plazo, modificando la oclusión, la fisiología bucal y afectando la calidad de vida. En la epidemiologia de los traumatismos dentales, en Hong Kong (18) un estudio realizado en sujetos con y $\sin$ TEA, mostro que los pacientes con TEA, tienen un porcentaje mayor de traumatismo dental en sector anterior (incisivos maxilares y caninos) aunque estos hallazgos no fueron estadísticamente significativos, coinciden con otro estudio en Brasil, de $2016^{(21)}$, donde los dientes más afectados fueron los incisivos maxilares permanentes. Acorde a la edad, un estudio realizado en Francia de $2019{ }^{(22)}$, evidenció una mayor presencia de traumatismos dentales en niños $(14,8 \%)$ en comparación a los pacientes mayores (adolescentes: 6,9\%; adultos maduros: 2,9\%), lo que podría estar relacionado con la diversidad de actividades y pasatiempos cotidianos en niños que suelen ser más riesgosos. En referencia al sexo, un estudio realizado en Brasil en $2016^{(21)}$ muestra que, en el grupo con TEA, la prevalencia de traumatismo dental fue mayor en mujeres $(41,7 \%)$ así como el riesgo de experimentarlo.

A la luz de los autores, la evidencia referente a los hallazgos extra orales, fue limitada, encontrando reportes enfocados en el crecimiento de la cabeza y cerebro, en caso de autismo severo que provoca variabilidad en el tamaño, velocidad y dirección de crecimiento ${ }^{(23)}$. Tema 
que escapa a los intereses propios de la presente revisión, por lo que no se realiza una descripción a mayor detalle.

\section{Consideraciones para la consulta odontológica, grupos de apoyo para fortalecer conductas de autocuidado y calidad de vida y salud bucal en sujetos con TEA}

Durante la atención odontológica, es necesario conocer las particularidades comportamentales asumidas por pacientes con TEA, ya que el trato odontólogo-paciente no se puede generalizar, sino que por el contrario requiere contextualizarse para ofrecer un servicio satisfactorio a las necesidades de pacientes y expectativas de padres y cuidadores. Para lograr este objetivo, incluso se sugiere previo a la consulta presencial, realizar un acercamiento mediante las tecnologías de la información y la comunicación, lo cual puede ayudar a minimizar posibles errores y adicionalmente permitirá obtener información estratégica referente a gustos y preferencias del ambiente, para abordar al potencial paciente y controlar factores tales como ruido, colores, distractores y aforo al interior del consultorio.

Es importante motivar la asistencia de estos pacientes a las citas preventivas y de control odontológico, dejando atrás el tradicional enfoque curativo enfocado en extracciones dentales y restauraciones ${ }^{(24)}$; sin embargo, existen ciertas dificultades de acceso para la atención odontológica (8), una de estas corresponde a los costos financieros, los cuales son significativamente mayores en quienes tienen TEA, un estudio en Estados Unidos de 2014 (25), mostró que las personas con TEA incurren en costos mayores para su atención. Otras dificultades expuestas, incluyen la escasez de cobertura para la asistencia (9), generando necesidades dentales insatisfechas (3), que impactan negativamente la calidad de vida relacionada con la salud bucal ${ }^{(1)}$ y que favorece el uso de servicios de urgencia ${ }^{(26)}$, lo que podría evitarse si se concediera el tratamiento oportuno requerido.

Existen pocos estudios respecto al uso de los servicios odontológicos en pacientes con TEA ${ }^{(11)}$. Algunos, se enfocan en las barreras de acceso para la atención, existiendo reportes que afirman que pese a que un alto porcentaje (97\%) visita la consulta odontológica, muchos pacientes no reciben el nivel requerido de atención ${ }^{(27)}$ e incluso, al comparar entre quienes presentan TEA y no, el uso de atención preventiva es inferior para los primeros ${ }^{(26)}$. Otro hallazgo, es la dificultad para encontrar odontólogos con las competencias, habilidades, vocación y conocimiento para trabajar con pacientes en situación de discapacidad ${ }^{(10)}$, sin embargo, se reconoce que algunas facultades de odontología vienen desarrollando esfuerzos e iniciativas para lograr sensibilizar a los estudiantes en referencia a la atención de pacientes en condiciones y con necesidades 
especiales, por lo que manera concienzuda, es menester revisar la necesidad de fortalecer estas acciones y extrapolarlas a los diversos programas de formación para mejorar los resultados en la atención. Del mismo modo, se enfatiza en la necesidad de que el recurso humano desarrolle habilidades sociales como la comprensión y calma para brindar atención odontológica, considerando los posibles comportamientos negativos que pueden presentar estos pacientes ${ }^{(28,29)}$. Esta situación se ilustra considerando por ejemplo lo reportado en una revisión de $2018{ }^{(17)}$, alusiva a los servicios de ortodoncia y al limitado acceso que tienen los pacientes con TEA para acceder a ortodontistas capacitados, convirtiéndose la atención en toda una complicación. Este hecho, puede quizás ser un indicio de lo que pudiera estar sucediendo en la consulta de odontología general y de otras especialidades.

En cuanto a las actitudes vocacionales requeridas, un estudio cualitativo enfocado en padres, empleando grupos focales, reportó que los odontólogos rechazan o se niegan para la atención de sujetos con TEA, debido a la dificultad para manejar el comportamiento de estos pacientes. Otras veces, los profesionales tergiversan lo concerniente a sus competencias y afirman que conocen como atender a esta población; sin embargo, la atención se convierte en un martirio para todos. Por esto, los padres afirman que, para contactar con odontólogos capacitados, requieren de voluntad e iniciativas propias para acercarse a otros padres de familia y por medio de referencias en internet, localizar a estos profesionales con experiencia para atención odontológica ${ }^{(30)}$. Por lo anterior, en la formación de pregrado de odontología, se recomienda promover empatía hacia el paciente y generar espacios que permitan ganar experiencia en la atención de población con necesidades especiales, favoreciendo la obtención de recurso humano cualificado para la atención de pacientes con TEA, un acceso comprometido y la adherencia a los tratamientos en los servicios odontológicos ${ }^{(31)}$.

Ahora bien, en la población con TEA, no existen perfiles específicos de comportamiento, por ende, no se puede predecir el desarrollo de la consulta odontológica, existiendo situaciones en las cuales puede existir total colaboración para realizar cualquier procedimiento $u$ otras, en donde no es posible realizar la valoración clínica ${ }^{(32)}$. Por esto, se recomienda que previo a la atención odontológica, se realice una cita de reconocimiento del caso y adecuación al clima de atención, para lograr una exposición incremental y controlada ante nuevos estímulos y contextos, garantizando mejor calidad en la atención y adaptación del paciente ${ }^{(33)}$, pues se ha documentado, que esta población puede existir un sistema nervioso con hipersensibilidad, respondiendo exageradamente a ciertos estímulos como sonidos específicos, luz, olores, texturas y toque o contacto ${ }^{(10)}$, o manifestar hiposensibilidad ante ciertos estímulos dolorosos y térmicos ${ }^{(3)}$, por lo que no es predecible la respuesta del paciente, pero sí realizar un sondeo 
preliminar de las respuestas ante el entorno y como interactúa el paciente con TEA. En este aspecto, un estudio realizado en Tailandia, en $2017^{(34)}$ en 95 pacientes con TEA ( 3 a 18 años de edad), valoró su cooperación durante la atención odontológica, mostrando que en esta influye la edad, la propiocepción, el comportamiento inicial en la consulta, la comunicación verbal, la capacidad de seguir instrucciones, el hábito de cepillado dental y el entrenamiento para ir al baño. Esta valiosa información se puede obtener durante la cita de adaptación mediante una amable anamnesis en compañía de padres y cuidadores.

Durante la atención odontológica, la existencia de comorbilidades como retraso del desarrollo y del habla, discapacidad intelectual y trastornos convulsivos ${ }^{(35)}$, condiciones bucales como alta actividad de caries, pueden afectar negativamente el curso de la atención odontológica, mientras el TEA grave y la discapacidad intelectual, se asocian con mayores necesidades dentales insatisfechas ${ }^{(36)}$. Caso contrario sucede con la edad avanzada, el funcionamiento cognitivo superior, existencia de habilidades de comunicación mayores y capacidad para realizar el autocuidado ${ }^{(27)}$.

En casos donde no es posible la cooperación para la intervención y quizás está presente el estrés derivado del procedimiento tanto en el paciente como en la familia, una alternativa es recurrir a la atención iniciando con la inhalación de óxido nitroso/oxígeno y finalmente la alternativa de uso de la anestesia general, permitiendo mejor planificación de los tratamientos y control de sus efectos ${ }^{(3)}$. Como se muestra con éxito en un estudio realizado en Francia de $2019^{(23)}$.

Los factores relacionados con el acceso a los servicios y el comportamiento de los sujetos con TEA, son orientadores hacia la necesidad de involucrar a padres y/o cuidadores durante la consulta para diagnóstico, la planificación y ejecución de los tratamientos, pues de esta manera se puede acceder a una información privilegiada para identificar necesidades particulares de cuidado bucal, como se afirma en un estudio cualitativo de $2015^{(37)}$, mediante 4 grupos focales conformados por padres de pacientes con TEA, quienes coinciden en que existe variabilidad para la tolerancia de los cuidados y tratamientos bucales entre los pacientes con TEA y como padres desean que sus hijos tengan una atención odontológica de calidad e individualizada considerando la opinión de padres y cuidadores, quienes al recibir información y capacitación como supervisores, podrían mejorar en el paciente con TEA su estado bucal y facilitar el desarrollo de procedimientos para alcanzar los resultados esperados $(8,11,19)$. En este sentido, la presencia de padres y/o cuidadores durante la consulta odontológica, permite ejercer control sobre la dinámica de la cita, al permitir una aproximación hacia la comprensión de la conducta y posibles reacciones de los pacientes con TEA, facilitando la identificación de la técnica de 
orientación conductual a implementar. Es crucial que, en la odontología, se realice una atención bucodental con enfoque familiar, pues la familia es una unidad de soporte y respaldo para la positiva respuesta y evolución de los pacientes ${ }^{(3)}$.

Para mejorar la experiencia de los pacientes con TEA durante la consulta odontológica, se han evaluado al interior de la consulta las situaciones molestas, situaciones calmantes y motivadoras, un estudio realizado en Turquía de $2018{ }^{(38)}$, muestra que para más de la mitad de los participantes, la principal situación molesta corresponde al uso de voz alta, seguido del contacto con desconocidos y ambientes muy concurridos, en tanto que las situaciones calmantes y motivadoras fueron la música, los juegos en Tablet y computador seguido de ver dibujos animados en televisión. Ratificando que el uso de herramientas visuales y auditivas mejora la comunicación en la consulta. En este sentido, es posible destacar el uso de una aplicación denominada Autistic Child Going to the Dentist (Niño autista yendo al dentista, traducido al español), para facilitar la comunicación entre pacientes autistas y profesionales de odontología, mostrando paso a paso los procedimientos y fases del tratamiento, mediante texto, imágenes y comentarios de audio, mostrando resultados satisfactorios en la disminución del número de citas preventivas y exámenes clínicos ${ }^{(39)}$. Otros esfuerzos exitosos, incluyen técnicas de modificación del comportamiento como la distracción visual utilizando gafas ${ }^{(40)}$, el uso de tarjetas con imágenes, tecnologías de video y aplicaciones móviles ${ }^{(41)}$, cuyo propósito es lograr la continua exposición a estímulos que permita la adaptación del paciente (citas de desensibilización) ${ }^{(3)}$.

Del mismo modo, se ha propuesto el uso de sistemas de comunicación por el intercambio de imágenes, en ingles se reconoce como The Picture Exchange Communication System (PECS), que ha sido adaptado para uso en odontología, favoreciendo la adaptación de pacientes con TEA durante tratamientos preventivos limitando el uso de restricción física ${ }^{(42)}$. Todas estas intervenciones, pertenecen a la pedagogía visual, aprovechando la capacidad que tienen los pacientes con TEA, para emitir una mejor respuesta ante imágenes en comparación al lenguaje verbal ${ }^{(3)}$. Existe una experiencia en Italia documentada en $2015^{\left({ }^{(4)}\right)}$, por medio de un enfoque multietapa, empleando soportes visuales para facilitar que los sujetos con TEA puedan someterse al examen clínico en sillón odontológico, recibir higiene profesional, sellado de fisuras y tratamiento restaurador, mostrando una tasa positiva de aceptación durante las etapas, lo que sugiere la necesidad de incursionar en el uso de estas herramientas desde la odontología para mejorar la atención. 
Otras experiencias, muestran un entorno odontológico adaptado sensorialmente para reducir angustia, incomodidad sensorial y percepción del dolor durante la profilaxis bucal, empleando la proyección de imágenes para generar distracción, la reproducción de música rítmica, el uso de un sillón odontológico con cubierta de mariposa para envolver al paciente, lo que ayuda a percibir un efecto calmante, tal como se muestra en un estudio piloto controlado aleatorizado realizado en 44 niños entre 6 y 12 años conformados por pacientes con TEA y sin TEA ${ }^{(44) .}$

Otras iniciativas incluyen programas para mejorar la comunicación entre los proveedores de salud, la familia y pacientes con TEA ${ }^{(45)}$, en donde involucrando al personal de atención y a la familia, se pueden mejorar los resultados de intervenciones y procedimientos y fortalecer las conductas de autocuidado. Lo cual se relaciona con que, las prácticas en salud proceden de las normas, objetivos, valores y comportamientos de los miembros de la familia/padres, si estos son positivos pueden favorecer hábitos de vida saludable en sus hijos con TEA ${ }^{(46)}$. Sin embargo, es preocupante la existencia limitada de estudios y programas para promover conocimiento, actitudes y comportamientos en padres respecto a salud bucal de sus hijos con TEA ${ }^{(29)}$. En este sentido, será importante sensibilizar a la comunidad académica, científica y asistencial, para la inclusión de padres y cuidadores dentro de sus planes, programas y políticas de enfoque educativo. Un estudio realizado en Egipto ${ }^{(29)}$, muestra un aumento en el número de respuestas correctas por parte de los padres que se someten a un programa educativo, lo que coincide con los resultados positivos de un estudio realizado en Hong Kong, en donde los padres de sujetos TEA mostraron mejor nivel de conocimiento y actitudes referentes al cuidado dental de sus hijos ${ }^{(47)}$.

Otro importante grupo identificado para brindar apoyo a sujetos con TEA, lo conforman los profesores, quienes comparten gran parte de la jornada cotidiana, y además aportan en su proceso de aprendizaje enfocado en la conducta de cuidado, como se evidencia en un estudio realizado en Singapur ${ }^{(48)}$, en donde un programa de capacitación dirigido a maestros, aumentó el nivel de conocimiento en salud bucal, de esta manera se logra beneficiar a los planes de estudio de instituciones dirigidas a población con necesidades especiales, en donde existen adicionalmente otros sujetos que se encuentran a cargo del cuidado y bienestar de personas con TEA. En un estudio realizado en Arabia Saudita de $2015^{(49)}$, para evaluar los conocimientos y actitudes que tenían proveedores de atención médica y educadores en lo referente a salud bucal de sujetos con TEA, se caracterizó al personal laboral, lo que permitió involucrar a todos los miembros del equipo. Adicionalmente el estudio mostró que casi la mitad de los participantes nunca ha aconsejado a padres de sujetos con TEA respecto a higiene bucal, más del $70 \%$ no ha referido a los niños a una consulta odontológica ni ha revisado los dientes de los 
niños, lo que quizás se debe a que no han recibido instrucciones de higiene bucal. Estas situaciones se tornan inquietantes y a su vez en un área de oportunidades para empezar a incluir a esta población en los planes y programas enfocados en salud bucal de sujetos con TEA, favoreciendo la transmisión de información fundamental y la adopción de medidas preventivas y de autocuidado por padres y las mismas personas con TEA.

El estado de salud bucal ha tomado relevancia, incluso en la percepción de calidad de vida, valorando la percepción que tienen los padres de sujetos con TEA referente a la calidad de vida relacionada con la salud bucal de sus hijos, evidenciándose que, aunque es mejor al compararse con sujetos sin TEA, persisten problemáticas sociales y de comunicación con esta población (50). Conociendo este antecedente, en el área de odontología debe existir interés por atender estos pacientes comprendiendo la importancia del establecimiento empatía y comunicación para que la atención en salud bucal impacte más allá de la cavidad bucal, en la salud general y percepción de calidad de vida en población con TEA y su familia.

\section{Conclusión}

El estado y progreso de enfermedades bucales como la caries dental, enfermedad periodontal y algunos hallazgos intraorales en sujetos con TEA, responde a condiciones particulares del síndrome y su tratamiento, constituyendo un gran desafío para la odontología, en la que se requieren profesionales cualificados y con vocación para desarrollar una atención odontológica empática y comprensiva en los cambios comportamentales que pueden presentar estos pacientes. Asimismo, se debe considerar la atención holística, considerando la condición de salud emocional y sensorial del TEA en la que se limita que estos sujetos asuman con total responsabilidad el cuidado de su cavidad bucal, requiriendo que las acciones sean dirigidas a los pacientes y quienes les rodean.

\section{Conflicto de interés}

Los autores declaran que no existe conflicto de interés con las instituciones centro de estudio participantes, que pudiera influir en los hallazgos reportados.

\section{Referencias}

1. da Silva SN, Gimenez T, Souza RC, Mello-Moura ACV, Raggio DP, Morimoto S, Lara JS, Soares GC, Tedesco TK. Oral health status of children and young adults with autism spectrum disorders: systematic review and meta-analysis. Int J Paediatr Dent. 2017 Sep;27(5):388-398. doi: 10.1111/ipd.12274. 
2. Pimienta N, González Y, Rodríguez L. Autismo infantil, manejo en la Especialidad de Odontología. Acta médica del centro. 2017; 11 (4):56-69.

3. Gandhi RP, Klein U. Autism spectrum disorders: an update on oral health management. J Evid Based Dent Pract. 2014 Jun; 14 Suppl:115-26. doi: 10.1016/j.jebdp.2014.03.002.

4. Brugha TS, Spiers N, Bankart J, et al. Epidemiology of autism in adults across age groups and ability levels. Br J Psychiatry. 2016;209(6):498-503. doi:10.1192/bjp.bp.115.174649.

5. Fombonne E. Editorial: The rising prevalence of autism. J Child Psychol Psychiatry. 2018;59(7):717-720. doi:10.1111/jcpp.12941.

6. Lyall K, Croen L, Daniels J, et al. The Changing Epidemiology of Autism Spectrum Disorders. Annu Rev Public Health. 2017; 38:81-102. doi:10.1146/annurev-publhealth031816-044318.

7. Morales-Hidalgo P, Roigé-Castellví J, Hernández-Martínez C, Voltas N, Canals J. Prevalence and Characteristics of Autism Spectrum Disorder Among Spanish SchoolAge Children. J Autism Dev Disord. 2018;48(9):3176-3190. doi:10.1007/s10803-0183581-2.

8. El Khatib AA, El Tekeya MM, El Tantawi MA, Omar T. Oral health status and behaviours of children with Autism Spectrum Disorder: a case-control study. Int J Paediatr Dent. 2014 Jul;24(4):314-23. doi: 10.1111/ipd.12067.

9. Naidoo $\mathrm{M}$, Singh S. The Oral health status of children with autism Spectrum disorder in KwaZulu-Nata, South Africa. BMC Oral Health. 2018 Oct 12;18(1):165. doi: 10.1186/s12903-018-0632-1.

10. Muraru D, Ciuhodaru T, \& Iorga M. Providing dental care for children with autism spectrum disorders. International Journal of Medical Dentistry. 2017; 7(2): 124-130.

11. Blomqvist M, Bejerot S, Dahllöf G. A cross-sectional study on oral health and dental care in intellectually able adults with autism spectrum disorder. BMC Oral Health. 2015; 15:81. Published 2015 Jul 15. doi:10.1186/s12903-015-0065-z.

12. Chan D, Chan S, So H, Li A, Ng R, \& Tsang N. Dental Health of Preschool Children with Autism Spectrum Disorder in Hong Kong. HK J Paediatr (new series) 2014; 19:161-168. 
13. Sarnat H, Samuel E, Ashkenazi-Alfasi N, Peretz B. Oral Health Characteristics of Preschool Children with Autistic Syndrome Disorder. J Clin Pediatr Dent. 2016 Winter; 40(1):21-5. doi: 10.17796/1053-4628-40.1.21.

14. Ninuk Hariyani, Roesanto Heroe Soebekti, Dini Setyowati, Taufan Bramantoro, Listyati Setyo Palupi, Oktarina,. and Ekky Putriana. Factors influencing the severity of dental caries among Indonesian children with autism spectrum disorder - a pilot study. Clin Cosmet Investig Dent. 2019; 11: 227-233.

15. Suhaib F, Saeed A, Gul H, Kaleem M. Oral assessment of children with autism spectrum disorder in Rawalpindi, Pakistan. Autism. 2019 Jan; 23(1):81-86. doi: 10.1177/1362361317730299.

16. Bartolomé-Villar B, Mourelle-Martínez MR, Diéguez-Pérez M, de Nova-García MJ. Incidence of oral health in paediatric patients with disabilities: Sensory disorders and autism spectrum disorder. Systematic review II. J Clin Exp Dent. (2016), doi:10.4317/ jced.52923.

17. Acharya S, Patnaik S, \& Nanda SB. "Patients with Autism Spectrum Disorders: Strategy for Orthodontic Care." Journal of Clinical \& Diagnostic Research Journal of Clinical and Diagnostic Research. 2018 Jul; 12(7): ZE04-ZE07. doi: 10.7860/JCDR/2018/32845.11761.

18. Du RY, Yiu CK, King NM, Wong VC, McGrath CP. Oral health among preschool children with autism spectrum disorders: A case-control study. Autism. 2015;19(6):746-751. doi:10.1177/1362361314553439.

19. Fakroon, S., Arheiam, A., \& Omar, S. Dental caries experience and periodontal treatment needs of children with autistic spectrum disorder. European Archives of Paediatric Dentistry. 2015; 16(2): 205-209.

20. Fontaine-Sylvestre C, Roy A1, Rizkallah J, Dabbagh B, Ferraz Dos Santos B. Prevalence of malocclusion in Canadian children with autism spectrum disorder. Am J Orthod Dentofacial Orthop. 2017 Jul; 152(1):38-41. doi: 10.1016/j.ajodo.2017.02.014.

21. Andrade NS, Dutra TT, Fernandes RF, Moita Neto JM, Mendes RF, Prado Júnior RR. Retrospective study of dental trauma in children with autism spectrum disorders: a paired study. Spec Care Dentist. 2016;36(5):260-264. doi:10.1111/scd.12180. 
22. Mangione F, Bdeoui F, Monnier-Da Costa A, Dursun E. Autistic patients: a retrospective study on their dental needs and the behavioural approach. Clin Oral Investig. 2020;24(5):1677-1685. doi:10.1007/s00784-019-03023-7.

23. Erazo C, Carrillo G, Velosa J. Caracterización de alteraciones craneofaciales en población con necesidades especiales: autismo y retardo mental. Revisión sistemática de la literatura. Universitas Odontológica. 2014; 33(71),19-39.

24. Mansoor D, Al Halabi M, Khamis AH, Kowash M. Oral health challenges facing Dubai children with Autism Spectrum Disorder at home and in accessing oral health care. European Journal of Paediatric Dentistry. 2018 Jun;19(2):127-133. DOI: 10.23804/ejpd.2018.19.02.06.

25. Nakao S, Scott JM, Masterson EE, Chi DL. Non-traumatic Dental Condition-Related Emergency Department Visits and Associated Costs for Children and Adults with Autism Spectrum Disorders. J Autism Dev Disord. 2015;45(5):1396-1407. doi:10.1007/s10803014-2298-0.

26. Chi DL, Momany ET, Mancl LA, Lindgren SD, Zinner SH, Steinman KJ. Dental Homes for Children With Autism: A Longitudinal Analysis of lowa Medicaid's I-Smile Program. Am J Prev Med. 2016 May;50(5):609-615. doi: 10.1016/j.amepre.2015.08.022.

27. Nelson T, Chim A, Sheller BL, McKinney CM, Scott JM. Predicting successful dental examinations for children with autism spectrum disorder in the context of a dental desensitization program. J Am Dent Assoc. 2017 Jul;148(7):485-492. doi: 10.1016/j.adaj.2017.03.015.

28. Stein Duker LI, Floríndez LI, Como DH, Tran CF, Henwood BF, Polido JC, Cermak SA. Strategies for Success: A Qualitative Study of Caregiver and Dentist Approaches to Improving Oral Care for Children with Autism. Pediatr Dent. 2019 Jan 15;41(1):4E-12E.

29. AbdAllah, E. A., N. E. Metwalli, and A. S. Badran. "Effectiveness of a one year oral health educational and preventive program in improving oral health knowledge and oral hygiene practices of a group of Autistic Egyptian children and their caregivers." Future Dental Journal.2018; 4 (1): 23-29.

30. Duker LIS, Henwood BF, Bluthenthal RN, Juhlin E1, Polido JC, Cermak SA. Parents' perceptions of dental care challenges in male children with autism spectrum disorder: An initial qualitative exploration. Res Autism Spectr Disord. 2017 Jul;39:63-72. doi: 10.1016/j.rasd.2017.03.002. 
31. Delli K, Reichart PA, Bornstein MM \& Livas C. Manejo de los niños con trastorno del espectro autista en el ámbito dental: Problemas, enfoques y recomendaciones de comportamiento. Medicina oral, patología oral y cirugía bucal. Ed. Española. 2014; 19(4): 233-240.

32. Posse JL, Novoa PC, Pazos M T. A, \& Barbosa IR. Aspectos conductuales de los pacientes con Trastorno del Espectro Autista (TEA) que condicionan su manejo en el ámbito odontológico. Medicina oral, patología oral y cirugía bucal. Ed. Española. 2014; 19(5): 288-293.

33. Nelson TM, Sheller B, Friedman CS, \& Bernier R. Educational and therapeutic behavioral approaches to providing dental care for patients with Autism Spectrum Disorder. Special Care in Dentistry. 2015; 35(3), 105-113.

34. Dangulavanich $\mathrm{W}$, Limsomwong $\mathrm{P}$, Mitrakul K, Asvanund $\mathrm{Y}$, Arunakul M. Factors associated with cooperative levels of Autism Spectrum Disorder children during dental treatments. Eur J Paediatr Dent. 2017;18(3):231-236. doi:10.23804/ejpd.2017.18.03.11.

35. Mathu-Muju KR, Li HF, Nam LH, Bush HM. Visualizing the Comorbidity Burden in Children with Autism Spectrum Disorder Receiving Dental Treatment Under General Anesthesia. Pediatr Dent. 2016; 38(2):134-139.

36. McKinney CM, Nelson T, Scott JM, Heaton LJ, Vaughn MG, Lewis CW. Predictors of unmet dental need in children with autism spectrum disorder: results from a national sample. Acad Pediatr. 2014 Nov-Dec;14(6):624-31. doi: 10.1016/j.acap.2014.06.023.

37. Lewis C, Vigo L, Novak L, Klein EJ. Listening to Parents: A Qualitative Look at the Dental and Oral Care Experiences of Children with Autism Spectrum Disorder. Pediatr Dent. 2015; 37(7):E98-E104.

38. Onol S, Kırzıoğlu Z. Evaluation of oral health status and influential factors in children with autism. Niger J Clin Pract. 2018;21(4):429-435. doi:10.4103/njcp.njcp_41_17.

39. Zink AG, Molina EC, Diniz MB, Santos MTBR, Guaré RO. Communication Application for Use During the First Dental Visit for Children and Adolescents with Autism Spectrum Disorders. Pediatr Dent. 2018 Jan 1;40(1):18-22. 
40. Fakhruddin KS, El Batawi HY. Effectiveness of audiovisual distraction in behavior modification during dental caries assessment and sealant placement in children with autism spectrum disorder. Dent Res J (Isfahan). 2017;14(3):177-182. doi:10.4103/17353327.208768.

41. Elmore JL, Bruhn AM, Bobzien JL. Interventions for the Reduction of Dental Anxiety and Corresponding Behavioral Deficits in Children with Autism Spectrum Disorder. J Dent Hyg. 2016;90(2):111-120.

42. Zink AG, Diniz MB, Rodrigues Dos Santos MT, Guaré RO. Use of a Picture Exchange Communication System for preventive procedures in individuals with autism spectrum disorder: pilot study. Spec Care Dentist. 2016;36(5):254-259. doi:10.1111/scd.12183.

43. Cagetti MG, Mastroberardino S, Campus S, et al. Dental care protocol based on visual supports for children with autism spectrum disorders. Med Oral Patol Oral Cir Bucal. 2015;20(5):e598-e604. Published 2015 Sep 1. doi:10.4317/medoral.20424.

44. Cermak SA, Stein Duker LI, Williams ME, Dawson ME, Lane CJ, Polido JC. Sensory Adapted Dental Environments to Enhance Oral Care for Children with Autism Spectrum Disorders: A Randomized Controlled Pilot Study. J Autism Dev Disord. 2015;45(9):28762888. doi:10.1007/s10803-015-2450-5.

45. Clark LA, Whitt S, Lyons K. Improving Communication Between Health Care Providers, Families, and Children with Autism Spectrum Disorder: The Linked Program. J Perianesth Nurs. 2019;34(5):889-899. doi:10.1016/j.jopan.2018.12.009.

46. Nonong $Y$, Setiawan A, Dewi F, \& Navaneetha C. "Oral health knowledge among parents of autistic child in Bandung-Indonesia." Dental Journal (Majalah Kedokteran Gigi) [Online]. 2014; 47 (3):146-152.

47. Du RY, Yiu CKY, King NM. Oral Health Behaviours of Preschool Children with Autism Spectrum Disorders and Their Barriers to Dental Care. J Autism Dev Disord. 2019 Feb;49(2):453-459. doi: 10.1007/s10803-018-3708-5.

48. Tong HJ, Lee HY, Lee YT, Low Y, Lim CR, Nair R. Factors influencing the inclusion of oral health education in individualized education plans of children with autism spectrum disorders in Singapore. Int J Paediatr Dent. 2017;27(4):255-263. doi:10.1111/ipd.12257. 
49. Murshid EZ. Dental knowledge of educators and healthcare providers working with children with autism spectrum disorders. Saudi Med J. 2015;36(12):1477-1485. doi:10.15537/smj.2015.12.12622.

50. Eslami N, Movahed T, Asadi M. Parents' Perceptions of the Oral Health-related Quality of Life of their Autistic Children in Iran. J Clin Pediatr Dent. 2018;42(6):422-426. doi:10.17796/1053-4625-42.6.3. 